hep-th/0306107

MCTP-03-28

PUPT-2088

\title{
Hadronic Density of States from String Theory
}

\author{
Leopoldo A. Pando Zayas ${ }^{1}$ and Diana Vaman ${ }^{2}$ \\ ${ }^{1}$ Michigan Center for Theoretical Physics, University of Michigan \\ Ann Arbor, MI 48109-1120 \\ lpandoz@umich.edu \\ ${ }^{2}$ Department of Physics, Princeton University \\ Princeton, NJ 08544 \\ dvaman@feynman.princeton. edu
}

\begin{abstract}
$\underline{\text { ABSTRACT }}$
Exactly soluble string theories describing a particular hadronic sector of certain confining gauge theories have been obtained recently as Penrose-Güven limits of the dual supergravity backgrounds. The effect of taking the Penrose-Güven limit on the gravity side translates, in the gauge theory side, into an effective truncation to hadrons of large $\mathrm{U}(1)$ charge (annulons). We present an exact calculation of the finite temperature partition function for the hadronic states corresponding to a Penrose-Güven limit of the Maldacena-Nùnez embedding of $\mathcal{N}=1$ SYM into string theory. It is established that the theory exhibits a Hagedorn density of states.

Motivated by this exact calculation we propose a semiclassical string approximation to the finite temperature partition function for confining gauge theories admitting a supergravity dual, by performing an expansion around classical solutions characterized by temporal windings. This semiclassical approximation reveals a hadronic energy density of states of Hagedorn type, with the coefficient determined by the gauge theory string tension as expected for confining theories. We argue that our proposal captures primarily information about states of pure $\mathcal{N}=1 \mathrm{SYM}$, given that this semiclassical approximation does not entail a projection onto states of large $\mathrm{U}(1)$ charge.
\end{abstract}




\section{Introduction}

The duality between $\mathcal{N}=4$ Supersymmetric Yang-Mills (SYM) theory and string theory in $A d S_{5} \times S^{5}$ [1] has given a concrete playground where ideas about the gauge/gravity correspondence have been widely tested. In a series of papers, some of them predating the AdS/CFT proposal, Klebanov and collaborators [2] established a direct relation between the entropy of $\mathcal{N}=4 \mathrm{SYM}$ and the Bekenstein Hawking entropy of a stack of near extremal D3-branes.

Attempts at generalizing the ideas of [2] to cases of supergravity backgrounds dual to confining gauge theories 3, 4] have encountered multiple obstacles. In particular, nonextremal generalizations of such supergravity backgrounds are conjectured to exists only for high enough temperatures after chiral symmetry is restored and the theory has settled into the deconfined phase. It is fair to say that the question of understanding the hadronic density of states has so far alluded a supergravity approach. An intuitive explanation for the failure of supergravity to capture the density of states of confining gauge theories can be given in terms of the string partition function. Identifying the string partition function with the gauge theory partition function, as instructed by the gauge/gravity correspondence, the standard genus expansion in string theory predicts the following form of the partition function:

$$
Z_{\text {string }}=N^{2} Z_{0}+N^{0} Z_{1}+\frac{1}{N^{2}} Z_{2}+\ldots
$$

where we identify $g_{s} \equiv N^{-1}$. This implies that to understand the confined phase (the $N^{0}$ term) one must consider strings with torus topology. From this point of view the deconfined quark-gluon contribution (the $N^{2}$ term) has been successfully understood at the supergravity level [2, 3, 4] via the Bekenstein Hawking entropy. In this paper we attempt to study the $Z_{1}$ term in the above expansion.

In principle, determining $Z_{1}$ requires knowledge of the full string spectrum. Although the full super string theory in AdS-like backgrounds remains a elusive goal, recently progress has been made by considering certain limits. In [5] a dictionary was established between certain large R-charge operators in $\mathcal{N}=4$ SYM and string theory in the Penrose limit of $A d S_{5} \times S^{5}$. In a somewhat generalizing proposal [6], it was argued that by way of studying classical configurations of the string sigma model in supergravity backgrounds one can obtain information about specific sectors of the spectrum of strings in such backgrounds. In particular, [6] provided a semiclassical string derivation of the anomalous dimension of twist-two operators in $\mathcal{N}=4 \mathrm{SYM}$. Interestingly, this relation holds for the corresponding twist-two operators in QCD. Other remarkable results, like the presence of hard amplitudes for strings, have been obtained in a conceptually similar line of attack which approximates string theory processes in AdS-like backgrounds by a convolution of wave functions in the AdS-like background and standard string theory amplitudes [7. This climate encourages us to looked for a semiclassical alternative to the computation of the finite temperature partition function.

The structure of $Z_{1}$ above turns out to be intimately related to the nature of the Hagedorn density of states in the gauge/gravity correspondence. Ever since in the 60's the analysis of experimental data from hadron scattering lead Hagedorn [8] to introduce the asymptotic 
bootstrap condition (now known as a Hagedorn density of states), the nature of this distribution has been a source of interest. In the 80's, explicit computations of the partition functions of all consistent string theories, showed the universality of the Hagedorn density of states in string theory. More recently, based on the solubility of string theory on a curved plane wave background with Ramond Ramond flux [9], a Hagedorn temperature has been established in a new exactly solvable string theory on a curved background with Ramond Ramond flux [10] (see also [11, 12, 13]). The universal appearance of a Hagedorn density of states in string theories adds to the fascination of the subject. Several authors have studied this very tantalizing similarity between gauge and string theories in the AdS/CFT context 14.

Our goal in this paper is twofold. First, we compute exactly the thermal partition function of a string theory description of certain hadronic states. By analyzing the structure of the result we are lead to a proposal which constitutes the second goal of this paper - a proposal for a semiclassical evaluation of the nonzero temperature partition function.

\section{A string theory dual to hadronic states}

In the framework of the AdS/CFT some supergravity solutions have been constructed that are dual to confining gauge theories. Most notable are the Klebanov-Strassler (KS) [15] and Maldacena-Núñez (MN) [16] solutions. Generalizing some of the ideas proposed in [5], a particular sector of these theories has been isolated. The resulting string theory is exactly solvable and describes hadronic excitations of the gauge theory [17. Since the Penrose-Güven limit of either KS or MN backgrounds entails boosting along a certain compact direction transverse to the gauge theory directions, the hadronic states that are dual to the string theory modes necessarily have large $U(1)$ charge. These hadronic states that are selected upon taking the Penrose-Güven limit may be viewed as ripples on an infinitely heavy configuration with a large $\mathrm{U}(1)$ charge, called annulon [17.

In this section we will consider the theory emerging as a limit of the Maldacena-Núñez solution since it is technically simpler to deal with, and since it already contains all of the features believed to be universal for duals of confining theories. The light-cone Hamiltonian in question is [17]:

$$
\begin{aligned}
H & =\frac{P_{i}^{2}}{2 p^{+}}+\frac{P_{4}^{2}}{2 p^{+}}+\frac{1}{2 \alpha^{\prime} p^{+}} \sum_{n=1}^{\infty} n\left(N_{n}^{i}+N_{n}^{4}\right) \\
& +\frac{1}{2 \alpha^{\prime} p^{+}} \sum_{n=0}^{\infty}\left(w_{n}^{a} N_{n}^{a}+w_{n}^{b} N_{n}^{b}\right) \\
& +\frac{1}{2 \alpha^{\prime} p^{+}} \sum_{n=0}^{\infty}\left(\omega_{n}^{\alpha} \mathcal{S}_{n}^{\alpha}+\omega_{n}^{\beta} \mathcal{S}_{n}^{\beta}\right) .
\end{aligned}
$$

where $i=1,2,3, a=5,6, b=7,8, \alpha=1,2,3,4$ and $\beta=5,6,7,8 ; N_{n}^{s}=a_{n}^{s, \dagger} a_{n}^{s}+\tilde{a}_{n}^{s, \dagger} \tilde{a}_{n}^{s}$ and $\mathcal{S}^{s}=S_{n}^{s, \dagger} S_{n}^{s}+\tilde{S}_{n}^{s, \dagger} \tilde{S}_{n}^{s}$ are bosonic and fermionic occupation numbers respectively which include both left and right movers. The frequencies that appear in the Hamiltonian are

$$
w_{n}^{a}=\sqrt{n^{2}+\left(m_{0} p^{+} \alpha^{\prime}\right)^{2}}, \quad w_{n}^{b}=\sqrt{n^{2}+\frac{1}{9}\left(m_{0} p^{+} \alpha^{\prime}\right)^{2}},
$$




$$
\omega_{n}^{\alpha}=\sqrt{n^{2}+\frac{1}{9}\left(m_{0} p^{+} \alpha^{\prime}\right)^{2}}, \quad \omega_{n}^{\beta}=\sqrt{n^{2}+\frac{4}{9}\left(m_{0} p^{+} \alpha^{\prime}\right)^{2}} .
$$

The general structure of the light-cone Hamiltonian is rather simple. In ten dimensions the bosonic sector contains eight physical degrees of freedom. Generically three of these eight are massless. These three fields can be traced to part of the Poincare symmetry in the original background. The remaining five degrees of freedom represent specific excitations of the ground state and they are generically massive bosons in two dimensions. Some of the values of the masses can be traced back to symmetries of the original background before taking the limit. In other words, the Hamiltonian receives contributions from the momentum and stringy excitations in the spatial directions of the field theory (index $i=1,2,3), H_{\|}$, and a contribution from the massive "zero" modes and excitations of the internal directions (index $s=4,5,6,7,8), H_{\perp}$.

There are two important features which both the bosonic MN and KS Hamiltonians share. First, both theories have the same $H_{\|}$. Second, they have two worldsheet bosons with mass $p^{+} \alpha^{\prime} m_{0}$. Where $m_{0}$ is defined in such a way that the energy $E$ of the string theory vacuum state is $J m_{0}$, where $J$ is a large number representing an internal $U(1)$ charge in the dual gauge theory. The two theories then share the fact that the lowest-lying mode of the two massive bosons shifts $E$ by exactly $m_{0}$ (see [17] for a more complete description and notation). There is a feature that was not completely understood in [17] and that is not believed to be universal: one of the transverse excitations is massless.

The expression (2.1) is, in principle, sufficient to calculate the thermal partition function. However, it is very convenient to use a path integral approach (see [10]). Following this approach we use that the building blocks of the full partition function are the partition function of a massive boson and a massive fermion on the torus described by the modular parameter $\tau$ :

$$
\begin{aligned}
z_{l c}^{(0,0)}\left(\tau, m_{0}\right) & =\left[\prod_{n_{1}, n_{2} \in \mathbf{Z}} \operatorname{Im} \tau\left(\left(\frac{2 \pi}{4 I m \tau}\right)^{2}\left|n_{1} \tau-n_{2}\right|^{2}+\frac{m_{0}^{2} \beta^{2}}{I m \tau^{2}}\right)\right]^{-1} \\
& =\exp \left[-\pi \operatorname{Im} \tau \sum_{n \in \mathbf{Z}} \sqrt{n^{2}+m^{2}}\right]\left[\prod_{n \in \mathbf{Z}}\left(1-\exp \left[2 \pi\left(-I m \tau \sqrt{n^{2}+m^{2}}+i \operatorname{Re} \tau\right)\right]\right)\right]^{-1} . \\
z_{l c}^{(0,1 / 2)}\left(\tau, m_{0}\right) & =\prod_{n_{1}, n_{2} \in \mathbf{Z}} \operatorname{Im\tau }\left(\left(\frac{2 \pi}{4 I m \tau}\right)^{2}\left|n_{1} \tau+\frac{2 n_{2}+1}{2}\right|^{2}+\frac{m_{0}^{2} \beta^{2}}{I m \tau^{2}}\right) \\
& =\exp \left[\pi \operatorname{Im} \tau \sum_{n \in \mathbf{Z}} \sqrt{n^{2}+m^{2}}\right] \prod_{n \in \mathbf{Z}}\left(1+\exp \left[2 \pi\left(-\operatorname{Im} \tau \sqrt{n^{2}+m^{2}}+i \operatorname{Re} \tau\right)\right]\right) .
\end{aligned}
$$

where $\operatorname{Im} \tau=\beta /\left(2 \pi p^{+}\right)$. The free energy of a gas of non-interacting strings is:

$$
F=-\frac{1}{\beta} \operatorname{Tr}\left[(-1)^{\mathbf{F}} \ln \left(1-(-1)^{\mathbf{F}}\right) e^{-\beta E}\right]
$$

We evaluate the above expression explicitly by series expanding the $\ln$ in and grouping the sum 
over even and odd integers:

$$
\begin{aligned}
F & =-\frac{1}{2 \pi l_{s}} \int_{0}^{\infty} \frac{d I m \tau}{I m \tau^{2}} \int_{-1 / 2}^{1 / 2} d \operatorname{Re} \tau\left\{\sum_{r=\mathrm{odd}} \exp \left(-\frac{\beta^{2} r^{2}}{2 \pi \alpha^{\prime} I m \tau}\right)\right. \\
& \times\left[I m \tau^{-1 / 2}|\eta(\tau)|^{-2}\right]^{4}\left[z_{l c}^{(0,0)}\left(\tau, \frac{m_{0} \beta r}{\operatorname{Im} \tau}\right)\right]^{2}\left[z_{l c}^{(0,0)}\left(\tau, \frac{m_{0} \beta r}{3 I m \tau}\right)\right]^{2} \\
& \times\left[z_{l c}^{(0,1 / 2)}\left(\tau, \frac{m_{0} \beta r}{3 I m \tau}\right)\right]^{4}\left[z_{l c}^{(0,1 / 2)}\left(\tau, \frac{2 m_{0} \beta r}{3 I m \tau}\right)\right]^{4} \\
& +\sum_{r=\text { even }}^{\prime} \exp \left(-\frac{\beta^{2} r^{2}}{2 \pi \alpha^{\prime} I m \tau}\right)\left[\operatorname{Im} \tau^{-1 / 2}|\eta(\tau)|^{-2}\right]^{4}\left[z_{l c}^{(0,0)}\left(\tau, \frac{m_{0} \beta r}{\operatorname{Im} \tau}\right)\right]^{2}\left[z_{l c}^{(0,0)}\left(\tau, \frac{m_{0} \beta r}{3 I m \tau}\right)\right]^{2} \\
& \left.\times\left[z_{l c}^{(0,0)}\left(\tau, \frac{m_{0} \beta r}{3 I m \tau}\right)\right]^{-4}\left[z_{l c}^{(0,0)}\left(\tau, \frac{2 m_{0} \beta r}{3 I m \tau}\right)\right]^{-4}\right\} .
\end{aligned}
$$

A simplifying way to look at the above expression (2.6) is to realize that it is nothing but the thermal one loop free energy of a string theory with the following content: four massless bosons, two bosons with mass $m_{0}$, two bosons with mass $m_{0} / 3$; four fermions with mass $m_{0} / 3$ and four more fermions with mass $2 m_{0} / 3$. Note the change in the spin structure of the fermionic contribution to the partition function which can be explained by the action of $(-1)^{\mathbf{F}}$.

From expression (2.6) and using some of the modular properties discussed in [10, we conclude that this string theory has a Hagedorn temperature given by the following equation:

$$
-\tilde{T}_{s} \beta_{H}^{2}+\frac{4}{3} \pi-8 \pi \gamma_{0}\left(m_{0} \beta_{H}\right)-8 \pi \gamma_{0}\left(\frac{m_{0}}{3} \beta_{H}\right)+16 \pi \gamma_{1 / 2}\left(\frac{m_{0}}{3} \beta_{H}\right)+16 \pi \gamma_{1 / 2}\left(\frac{2 m_{0}}{3} \beta_{H}\right)=0,
$$

where $\gamma_{0}(M)=\sum_{n \in \mathbf{Z}} \sqrt{n^{2}+M^{2}}$ is the Casimir energy of a massive bosonic degree of freedom, while $\gamma_{1 / 2}(M)=\sum_{n \in \mathbf{Z}+1 / 2} \sqrt{n^{2}+M^{2}}$ is the Casimir energy of a fermionic degree of freedom, with spin structure $(1 / 2,0)$. In the previous relation we have used the mapping between string theory and gauge theory quantities obtained in [17], in particular $1 / 2 \pi \alpha^{\prime}=T_{s} / J=\tilde{T}_{s}$. The generalization of the Penrose-Güven limit taken in [17] amounts, on the gauge theory side, to sending the string tension and the $U(1)$ charge of the state to infinity: $T_{s}, J \rightarrow \infty$ with the ratio $\tilde{T}_{s}=T_{s} / J$ held fixed.

Notice that for small $m_{0}$ the value of the Hagedorn temperature reduces to that of IIB strings in flat space. For any nonvanishing $m_{0}$ we find an increase in the value of the Hagedorn temperature.

More important is the regime of very large $m_{0}$ since it has proved to be very relevant in gauge theory applications of the BMN construction. As shown in [10] for very large values of $m_{0}$ the functions $\gamma_{0}$ and $\gamma_{1 / 2}$ exponentially vanish and the main contribution comes, exclusively, from the directions where the hadrons can scatter, that is, the flat directions. The density of states in the region $\left(m_{0} \rightarrow \infty\right)$ is thus

$$
d(E) \approx \exp \left(\sqrt{\frac{4 \pi}{3}} \frac{E}{\tilde{T}_{s}^{1 / 2}}\right)
$$


This is in contrast to the situation for the plane wave [10, 11, 12] where the Hagedorn temperature goes to infinity in this limit. It can be shown that the Hagedorn temperature is a monotonic function of $m_{0}$ [12]. Thus, as we vary $m_{0}$ the Hagedorn temperature range is $\tilde{T}_{s}^{1 / 2} / \sqrt{4 \pi} \leq T_{H} \leq \sqrt{3} \tilde{T}_{s}^{1 / 2} / \sqrt{4 \pi}$.

\section{The role of temporal windings}

There are various effective ways in string theory to think about the Hagedorn temperature. It was realized by Polchinski [18] that the standard field theoretic approach of compactifying time to study thermal properties of field theory could be extended to string theory as well, by simply compactifying the target space time. This opens up the possibility of interpreting the Hagedorn density of states as caused by the windings in the temporal direction. This development was extended in [19] and further elaborated upon in [20].

Let us first, for completeness, recall the structure of the partition function for a compactified boson on the torus [21]. We choose the metric with torus topology to be

$$
d s^{2}=\left|d \sigma_{1}+\tau d \sigma_{2}\right|^{2}=d \sigma_{1}^{2}+|\tau|^{2} d \sigma_{2}^{2}+2(\operatorname{Re} \tau) d \sigma_{1} d \sigma_{2} .
$$

The worldsheet action for a bosonic field is

$$
S=\frac{1}{4 \pi \alpha^{\prime}} \int d \sigma_{1} d \sigma_{2} \sqrt{\gamma} \gamma^{\alpha \beta} \partial_{\alpha} X \partial_{\beta} X
$$

We are interested in considering configurations with nonzero winding number and therefore consider

$$
\begin{aligned}
X & =X_{m, n}^{\text {classical }}+X^{\text {quantum }} \\
X_{m, n}^{\text {classical }} & =m \beta \sigma_{1}+n \beta \sigma_{2} \\
X^{\text {quantum }} & =\sum_{n_{1}, n_{2}} X_{n_{1}, n_{2}} e^{2 \pi i\left(n_{1} \sigma_{1}+n_{2} \sigma_{2}\right)} .
\end{aligned}
$$

Note that $X^{\text {classical }}$ satisfies the equation of motion $\partial_{\alpha}\left(\sqrt{\gamma} \gamma^{\alpha \beta} \partial_{b} X\right)=0$, and determines a topological sector $(m, n)$; hence the notation. The quantum part is single-valued and corresponds precisely to the expansion of a bosonic field on the torus. Evaluating the action on the classical configuration we have

$$
S\left[X_{m, n}^{\text {classical }}\right]=\beta^{2} \frac{|m \tau-n|^{2}}{4 \pi \alpha^{\prime} I m \tau} .
$$

In calculating the partition function, the part coming from the single-valued $X^{\text {quantum }}$ is modular invariant by itself. To render the full partition function modular invariant we need to sum over all pairs $(m, n) \in \mathbf{Z}^{2}$. The free energy written in a manifestly modular invariant way is ${ }^{1}$

\footnotetext{
${ }^{1}$ Including the sum over $(m, n) \in \mathbf{Z}^{2}$ for modular invariance amounts effectively to account for multi-string states which is nothing but taking the logarithm of the partition function of a noninteracting string gas.
} 


$$
\begin{aligned}
F & =-\frac{1}{\beta} Z_{T^{2}} \\
& =-\frac{1}{2 \pi l_{s}} \sum_{m, n \in \mathbf{Z}}{ }^{\prime} \int_{\mathcal{F}} \frac{d^{2} \tau}{I m \tau^{2}} \frac{1}{I m \tau^{1 / 2}|\eta(\tau)|^{2}} \sum_{m, n \in \mathbf{Z}}{ }^{\prime} \exp \left(-\frac{\beta^{2}}{4 \pi \alpha^{\prime} I m \tau}|m \tau-n|^{2}\right) .
\end{aligned}
$$

Note that in this case the free energy factorizes as

$$
F=F_{\text {quantum }} F_{\text {classical }}
$$

This factorization turns out to be a property of flat space but the idea of computing the full partition function by first finding a classical configuration which incorporates the temporal windings, and then considering quantum fluctuations around it will be central to our proposal for computing the thermal partition function semiclassically.

At this point we are ready to revisit the partition function of the annulons in the previous section. Our intention is to cast the result of section 2 in a way that allows an interpretation as the partition function calculated based on a classical solution and quantum fluctuations around it. There are, however, a few subtleties to take into account. The natural candidate for a classical solution would be the temporal coordinate playing the role of a compactified boson described above. However, in the light-cone treatment of section 2 the time target space coordinate is gauged away. A fully covariant approach requires dealing with the RR 3-form field. We are content though with a partially covariant approach, in the sense that the bosons are treated covariantly, while the fermions are $\kappa$ gauge fixed.

To make explicit the emergence of a semiclassical solitonic configuration, it is crucial to note that although we have suggestively written the integration variables as $\operatorname{Re} \tau$ and $\operatorname{Im} \tau$, the partition function (2.6) has no obvious modular properties since we are integrating over the strip

$$
E: \quad \operatorname{Im} \tau>0, \quad-\frac{1}{2}<\operatorname{Re} \tau<\frac{1}{2} .
$$

An interesting result obtained for strings in flat space [22] but that can be generalized to the current situation is that the torus partition function (2.6] and 3.5) can be written in an explicitly modular invariant way:

$$
\begin{aligned}
Z_{T^{2}} & =\frac{\beta}{2 \pi l_{s}} \int_{\mathcal{F}} \frac{d I m \tau}{\operatorname{Im} \tau^{2}} \int d \operatorname{Re} \tau \sum_{m, n}{ }^{\prime} \exp \left(-\frac{\beta^{2}|m \tau-n|^{2}}{4 \pi \alpha^{\prime} \operatorname{Im} \tau}\right) \\
& \times\left[I m \tau^{-1 / 2}|\eta(\tau)|^{-2}\right]^{4}\left[z_{l c}^{(0,0)}\left(\tau, \frac{m_{0} \beta}{\operatorname{Im} \tau}|m \tau-n|\right)\right]^{2}\left[z_{l c}^{(0,0)}\left(\tau, \frac{m_{0} \beta}{3 \operatorname{Im} \tau}|m \tau-n|\right)\right]^{2} \\
& \times\left[z_{l c}^{(b 1, b 2)}\left(\tau, \frac{m_{0} \beta}{3 I m \tau}|m \tau-n|\right)\right]^{4}\left[z_{l c}^{\left(b_{1}, b_{2}\right)}\left(\tau, \frac{2 m_{0} \beta}{3 \operatorname{Im} \tau}|m \tau-n|\right)\right]^{4}
\end{aligned}
$$

where $m$ and $n$ are integers and we exclude $m=n=0$; also $b_{1}=\left(1-(-1)^{m}\right) / 4, b_{2}=$ $\left(1-(-1)^{n}\right) / 4$ denote the fermion spin structure in a given topological sector. Notice that the 
integration is over the fundamental domain

$$
\mathcal{F}: \quad|\tau|>1, \quad-\frac{1}{2}<\operatorname{Re} \tau<\frac{1}{2} .
$$

The proof of the above presentation of the partition function relies on the definition of fundamental domain: a region of the upper half-plane such that no two points are related by a modular transformation and any point outside of it can be reached by a modular transformation. Using this definition one is to write the strip in terms of the fundamental domain $\mathcal{F}$.

It is the exponent in the first line above (3.8) that can be interpreted as the classical action of temporal winding modes. The most important result of this manipulation is the explicit possibility of interpreting the partition function of a string theory in a curved background with RR form field as obtained from quantum fluctuations around a classical solution which involve windings of the spacetime temporal direction.

One interesting observation is that we see that the total partition function no longer factorizes as the product of $Z_{\text {quantum }}$ and $Z_{\text {classical }}$, confirming that this factorization was indeed an artifact of flat space. Nevertheless, the dependence of $Z_{\text {quantum }}$ on $(m, n)$ is rather simple, it reduces to:

$$
m_{0} \rightarrow m_{0}|m \tau-n|
$$

\section{A semiclassical evaluation of $Z_{1}$}

Let us assume that we have a supergravity background dual in the AdS/CFT sense to a gauge theory. The full string theory in such backgrounds is not known. However, for a semiclassical treatment the sigma model action is needed only up to quadratic terms and it is given by (we follow [23]):

$$
\begin{aligned}
S & =\frac{1}{4 \pi \alpha^{\prime}} \int d \sigma_{1} d \sigma_{2} \sqrt{\gamma}\left[\left(g_{\mu \nu} \gamma^{\alpha \beta}+b_{\mu \nu} \epsilon^{\alpha \beta}\right) \partial_{\alpha} X^{\mu} \partial_{\beta} X^{\nu}\right. \\
& \left.+i\left(\gamma^{\alpha \beta} \delta_{I J}-\epsilon^{\alpha \beta}\left(\rho_{3}\right)_{I J}\right) \partial_{\alpha} X^{m} \bar{\theta}^{I} \Gamma_{m} D_{\beta} \theta^{J}\right]
\end{aligned}
$$

where $\theta^{I} \quad(I=1,2)$ are the two real positive chirality 10-d MW spinors and $D_{b}$ is the pullback to the world-sheet of the supergravity covariant derivative in the variation of the gravitino :

$$
D_{\alpha}=\partial_{\alpha}+\frac{1}{4} \partial_{\alpha} X^{m}\left[\left(\omega_{\mu \nu m}-\frac{1}{2} H_{\mu \nu m} \rho_{3}\right) \Gamma^{\mu \nu}+\left(\frac{1}{3 !} F_{\mu \nu \lambda} \Gamma^{\mu \nu \lambda} \rho_{1}+\frac{1}{2 \cdot 5 !} F_{\mu \nu \lambda \rho \kappa} \Gamma^{\mu \nu \lambda \rho \kappa} \rho_{0}\right) \Gamma_{m}\right]
$$

where the $\rho_{s}$-matrices in the $I, J$ space are the Pauli matrices $\rho_{1}=\sigma_{1}, \rho_{0}=i \sigma_{2}, \rho_{3}=\sigma_{3}$.

The basic idea for a semiclassical estimation of the nonzero temperature partition function is schematically as follows. We consider the existence of winding temporal modes a crucial ingredient and thus, include them as part of the ansatz. In general, due to the nontriviality of 
the warp factor $\left(g_{00}\right)$, this ansatz will fail and the "minimal" modification one is to consider as the classical solution is

$$
X^{0}=m \beta \sigma_{1}+n \beta \sigma_{2}, \quad r=r\left(\sigma_{1}, \sigma_{2}\right) .
$$

Assuming that the background contains only a nontrivial metric and RR 3-form (for simplicity we are keeping in mind the solution of [16]), the nontrivial equations of motions are:

$$
\begin{aligned}
\partial_{\alpha}\left(\sqrt{\gamma} \gamma^{\alpha \beta} g_{00} \partial_{\beta} X^{0}\right) & =0 \\
\partial_{\alpha}\left(\sqrt{\gamma} \gamma^{\alpha \beta} g_{r r} \partial_{\beta} r\right)-\frac{1}{2} \partial_{r} g_{00}\left[\sqrt{\gamma} \gamma^{\alpha \beta} \partial_{\alpha} X^{0} \partial_{\beta} X^{0}\right] & =0 .
\end{aligned}
$$

Given a classical solution $\left(X^{0}, r\right)$, the action can be evaluated as:

$$
\begin{aligned}
S\left[X_{m, n}^{\text {classical }}\right] & =\frac{1}{4 \pi \alpha^{\prime}} \int d \sigma_{1} d \sigma_{2} \frac{1}{\operatorname{Im} \tau}\left[\beta^{2}\left(n^{2}+m^{2}|\tau|^{2}-2(\operatorname{Re} \tau) m n\right) g_{00}\right. \\
& \left.+g_{r r}\left(|\tau|^{2} \dot{r}^{2}+r^{\prime 2}-2(\operatorname{Re}) \dot{r} r^{\prime}\right)\right] .
\end{aligned}
$$

The solution to the coupled system of differential equations (4.4) is in general very involved. There is, however, a case were a simple solution exists. Let us assume the existence of a point $r_{0}$ such that:

$$
\exists r_{0}: g_{00}\left(r_{0}\right) \neq 0, \quad \partial_{r} g_{00}\left(r_{0}\right)=0, \quad \partial_{r} g_{r r}\left(r_{0}\right)=0 .
$$

Then, one solution to the system (4.4) is simply

$$
X^{0}=m \beta \sigma_{1}+n \beta \sigma_{2}, \quad r\left(\sigma_{1}, \sigma_{2}\right)=r_{0} .
$$

Now the question is - what kind of supergravity backgrounds in the context of the AdS/CFT admit such behavior? In a series of papers Sonnenschein and collaborators 24 have shown that the conditions (4.6) are precisely the conditions the supergravity background must satisfy in order for the dual gauge theory to be confining. This analysis is based on the AdS/CFT evaluation of the expectation value of the Wilson loop introduced in [25. Moreover, the tension in the gauge theory dual is given by $T_{s}=g_{00}\left(r_{0}\right) / 2 \pi \alpha^{\prime}$. Thus, a solution of the type (4.7) exists for any supergravity background dual to a confining theory. The classical action then becomes

$$
S\left[X_{\text {classical }}^{0}, r=r_{0}\right]=T_{s} \frac{\beta^{2}|m \tau-n|^{2}}{2 \operatorname{Im} \tau} .
$$

The most salient feature of this classical action is that the effective string tension that appears is precisely that of the confining gauge theory.

\subsection{Fluctuations}

Let us now turn to the evaluation of $Z_{\text {quantum }}$ by considering quadratic fluctuations around the classical solution (4.7). For simplicity and concreteness we will consider the background 
describing $N$ D5-branes wrapped on $S^{2}$ [16, 26] but the method applies to any supergravity dual to a confining gauge theory.

The metric of the solution is

$$
\begin{aligned}
d s^{2} & =e^{\phi}\left[d x^{a} d x_{a}+\alpha^{\prime} g_{s} N\left(d \tau^{2}+e^{2 g(\tau)}\left(e_{1}^{2}+e_{2}^{2}\right)+\frac{1}{4}\left(e_{3}^{2}+e_{4}^{2}+e_{5}^{2}\right)\right],\right. \\
e^{2 \phi} & =e^{-2 \phi_{0}} \frac{\sinh 2 \tau}{2 e^{g(\tau)}} \\
e^{2 g(\tau)} & =\tau \operatorname{coth} 2 \tau-\frac{\tau^{2}}{\sinh ^{2} 2 \tau}-\frac{1}{4},
\end{aligned}
$$

where,

$$
\begin{aligned}
& e_{1}=d \theta_{1}, \quad e_{2}=\sin \theta_{1} d \phi_{1}, \\
& e_{3}=\cos \psi d \theta_{2}+\sin \psi \sin \theta_{2} d \phi_{2}-a(\tau) d \theta_{1}, \\
& e_{4}=-\sin \psi d \theta_{2}+\cos \psi \sin \theta_{2} d \phi_{2}-a(\tau) \sin \theta_{1} d \phi_{1}, \\
& e_{5}=d \psi+\cos \theta_{2} d \phi_{2}-\cos \theta_{1} d \phi_{1}, \quad a(\tau)=\frac{\tau^{2}}{\sinh ^{2} \tau} .
\end{aligned}
$$

The position referred to as $r_{0}$ in the previous subsection is $\tau=0$. Therefore, we will expand the metric around that value. A fairly nontrivial fact we use is that $e_{3}^{2}+e_{4}^{2}+e_{5}^{2}$ evaluated at $\tau=0$ is the round $S^{3}$ with radius $1 / \sqrt{2}$. This can be verified by writing this line element as the $S U(2)$ invariant line element via a direct map involving a specific $S U(2)$ matrix [27. Therefore, we parametrize this round $S^{3}(\theta, \phi, \psi)$ by its Euler angles. Next, choosing to expand near the classical value $\theta=\pi / 2$ leads us effectively to $\mathbf{R}^{3}\left(y^{1}, y^{2}, y^{3}\right)$. Near $\tau=0$ we have that $e^{2 g} \approx \tau^{2}$ and therefore we combine the $\tau$-direction with $S^{2}\left(\theta_{1}, \phi_{1}\right)$ into $\mathbf{R}^{3}\left(\tau^{1}, \tau^{2}, \tau^{3}\right)$ in Cartesian coordinates. The end result for the bosonic quadratic action is

$$
\begin{aligned}
S_{2 b} & =S\left[X_{\text {classical }}^{0}, r=r_{0}\right]+\frac{1}{2 \pi \alpha^{\prime}} \int d \sigma_{1} d \sigma_{2} \sqrt{\gamma} \gamma^{\alpha \beta}\left(\partial_{\alpha} X^{a} \partial_{\beta} X^{a} g_{00}\right. \\
& \left.+\alpha^{\prime} g_{s} N g_{00}\left[\partial_{\alpha} \tau^{i} \partial_{\beta} \tau_{i}+\frac{1}{4} \partial_{\alpha} y^{i} \partial_{\beta} y_{i}\right]+\frac{4 \beta^{2}}{9 I m \tau^{2}} g_{00}|m \tau-n|^{2} \tau^{i} \tau_{i}\right)
\end{aligned}
$$

where $a=1, \ldots, 4$ and $i=1,2,3$.

To read off the mass term generated in the $\tau_{i}$ directions, we must first rescale the fields $\tau_{i}$ such that the kinetic term is canonically normalized. The bosonic part of the action yields seven massless fields, three massive fields with mass $(2 / 3) \beta \sqrt{\frac{1}{\alpha^{\prime} g_{s} N}}|m \tau-n| / \operatorname{Im} \tau$, and two diffeomorphism ghosts. When evaluating the one loop partition function, the ghost contribution will cancel out the contribution of two of the massless fluctuations, leaving us with three massive and four massless bosonic physical degrees of freedom.

Let us turn now to the fermionic degrees of freedom. The part quadratic in fermions can be expanded in the presence of a RR 3-form field strength and around (4.7) to give:

$$
S_{2 f}=\frac{i}{2 \pi \alpha^{\prime}} \int \bar{\theta}^{I}\left(\sqrt{\gamma} \gamma^{\alpha \beta} \delta^{I J}-\epsilon^{\alpha \beta} \sigma_{3}^{I J}\right) \partial_{\alpha} X^{0} \Gamma_{\underline{0}} e^{\frac{0}{0}}\left(\delta^{J K} \partial_{\beta}+\frac{1}{8 \cdot 3 !} e^{\phi} \sigma_{1}^{J K} \Gamma^{\mu_{1} \mu_{2} \mu_{3}} F_{\mu_{1} \mu_{2} \mu_{3}} \partial_{\beta} X^{0} \Gamma_{\underline{0}} e^{\underline{0}}\right) \theta^{K}
$$


where the 3 -form is given by $F_{(3)}=-\frac{1}{4} g_{s} N d y_{1} \wedge d y_{2} \wedge d y_{3}$ and we denoted the vielbeins by $e \frac{m}{\mu}$ : $e \frac{m}{\mu} e \frac{n}{\nu} \eta_{\underline{m n}}=g_{\mu \nu}$.

This action is still invariant under $\kappa$ symmetry. To truncate to the physical degrees of freedom, we choose the $\kappa$ gauge

$$
\Gamma^{+} \theta^{I}=0
$$

Following the analysis of [28] one is left then with a theory which is free of conformal anomaly ${ }^{2}$.

Then the $\kappa$ gauge fixed action $S_{2 f}$ becomes

$$
\begin{aligned}
& S_{2 f}=\frac{i}{2 \pi \alpha^{\prime}} \int \quad \bar{\theta}^{I}\left(\sqrt{\gamma} \gamma^{\alpha \beta} \delta^{I J}-\epsilon^{\alpha \beta} \sigma_{3}^{I J}\right) \partial_{\alpha} X^{+} \Gamma^{-}\left(g_{00}\right)^{-1 / 2}\left(\delta^{J K} \partial_{\beta}\right. \\
& \left.+\frac{1}{8 \cdot 3 !} e^{\phi} \sigma_{1}^{J K} \Gamma_{\underline{i}_{1}^{\prime} \underline{i}_{2}^{\prime} \underline{i}_{3}^{\prime}} F_{i_{1}^{\prime} i_{2}^{\prime} i_{3}^{\prime}} \partial_{\beta} X^{+} \Gamma^{-}\left(g_{00}\right)^{-1 / 2}\right) \theta^{K}
\end{aligned}
$$

where we have used that the classical solution is characterized by non-vanishing $X^{ \pm}$as well as the gauge condition. Next notice that the term proportional to $F_{(3)}$ cancels by the gauge condition. We are left therefore with 8 massless GS fermions.

One last ingredient needed for the finite temperature partition function relates to the fact that in the path integral, the boundary conditions obeyed by the thermalized fermionic degrees of freedom in a given soliton sector, characterized by $(m, n)$ winding numbers, are [29]

$$
\begin{aligned}
& \theta\left(\sigma_{1}+1, \sigma_{2}\right)=(-1)^{m} \theta\left(\sigma_{1}, \sigma_{1}\right) \\
& \theta\left(\sigma_{1}, \sigma_{2}+1\right)=(-1)^{n} \theta\left(\sigma_{1}, \sigma_{1}\right) .
\end{aligned}
$$

Finally, putting all the pieces together, the one loop finite temperature partition function will be given by

$Z_{T^{2}}=\sum_{m, n \in \mathbf{Z}}{ }^{\prime} \frac{\beta}{2 \pi l_{s}} \int_{\mathcal{F}} d^{2} \tau \frac{1}{I m \tau^{2}} e^{-\frac{\beta^{2} g_{00}}{4 \pi \alpha^{\prime}} \frac{|m \tau-n|^{2}}{I m \tau}} z_{0,0}^{b}(\tau, 0)^{5} z_{0,0}^{b}\left(\tau, M^{2}=\frac{4}{9} \beta^{2} \frac{|m \tau-n|^{2}}{I m \tau^{2}} \frac{1}{\alpha^{\prime} g_{s} N}\right)^{3} z_{b_{1}, b_{2}}^{f}(\tau, 0)^{8}$

where [10]

$$
z_{0,0}^{b}(\tau, M)=e^{-\pi I m \tau \sum_{l \in \mathbf{Z}} \sqrt{l^{2}+M^{2}}} \prod_{l \in \mathbf{Z}}\left(1-e^{-2 \pi I m \tau \sqrt{l^{2}+M^{2}}+2 \pi i R e \tau l}\right)^{-1}
$$

denotes the contribution of a bosonic degree of freedom with mass $M$ and regular boundary conditions, while

$$
z_{b_{1}, b_{2}}^{f}(\tau, M)=e^{\pi I m \tau \sum_{l \in \mathbf{Z}} \sqrt{\left(l+b_{1}\right)^{2}+M^{2}}} \prod_{l \in \mathbf{Z}}\left(1-e^{-2 \pi I m \tau \sqrt{\left(l+b_{1}\right)^{2}+M^{2}}+2 \pi i \operatorname{Re} \tau\left(l+b_{1}\right)-2 \pi i b_{2}}\right),
$$

\footnotetext{
${ }^{2}$ It was noted in 28] that the conformal anomaly vanishes for the GS string in the $\kappa$ gauge in a flat target space background, where the analysis reduces to considering the central charge contribution of the ten scalars, one pair of ghosts and eight pairs of GS fermions: $10-26+8 \times 4 \times \frac{1}{2}=0$. The contribution to the conformal anomaly of a GS fermion pair is 4 times that of a 2d Majorana worldsheet spinor [28]. More generally, in curved backgrounds, the vanishing of the conformal anomaly is proven by computing the effective Liouville action.
} 
denotes the contribution of a GS fermion, with mass $M$ and in the soliton sector $m, n$, with twisted boundary conditions $b_{1}=\left(1-(-1)^{m}\right) / 2$ and $b_{2}=\left(1-(-1)^{n}\right) / 2$.

The partition function (4.16) has a potential divergence as $\operatorname{Im} \tau \rightarrow \infty$. Reading off only the dominant exponentials in this limit we find that for $m=1$ the integrand of the partition function

$$
Z_{T^{2}} \approx \int e^{-\frac{\beta^{2} g_{00}}{4 \pi \alpha^{\prime}} \operatorname{Im} \tau} e^{-\pi I m \tau \sum_{l \in \mathbf{Z}}\left(5 l+3 \sqrt{l^{2}+\frac{4}{9} \beta^{2} \frac{1}{\alpha^{\prime} g_{s} N}}-8\left(l+\frac{1}{2}\right)\right)},
$$

becomes divergent at a critical temperature $T_{H}$ :

$$
\frac{1}{4 \pi \alpha^{\prime}} \beta_{H}^{2} g_{00}=-2 \pi\left(5 \gamma_{0}(0)+3 \gamma_{0}\left(2 \beta_{H} \sqrt{\frac{1}{\alpha^{\prime} g_{s} N}} / 3\right)-8 \gamma_{1 / 2}(0)\right) .
$$

The Hagedorn temperature is none other than the temperature where the first winding soliton becomes tachyonic [20].

In the limit of very large $1 /\left(\alpha^{\prime} g_{s} N\right)$ we find that the density of states is given by

$$
d(E) \approx \exp \left(\sqrt{3 \pi} \frac{E}{T_{s}^{1 / 2}}\right) .
$$

Note that the density of states depends, as advertised, on the gauge theory quark-antiquark string tension.

\section{Conclusions}

In our proposal there is certainly an ambiguity in the choice of the classical solution around which to expand. We have argued for the need for temporal winding modes but this does not exclude other configurations. In fact, in principle the partition function evaluated semiclassically should include a sum over all classical solutions. Effectively, in this paper we have considered two such expansions. The annulons are precisely an expansion around a classical solution which corresponds to large $U(1)$ flavor charge in the field theory. The advantage of the simple choice advocated in the second part of the paper is precisely the absence of any charge, since in order to hope to collect information close to the pure $\mathcal{N}=1$ sector which is neutral under any charge contained in the supergravity background we should not involve any charge. The only possible charge $-U(1)_{R}$ - is broken by gaugino condensate in the IR. Thus, our choice of the classical solution is justified for the purpose of extracting information about pure $\mathcal{N}=1$ SYM.

Let us comment on the nature of the density of states discussed here. The "confinement/deconfinement" of $\mathcal{N}=4 \mathrm{SYM}$ on $S^{3}$ is kinematical, reflecting just the $N^{0}$ versus $N^{2}$ transition in the free energy [30]. Similarly, properties like a Hagedorn temperature in these conformal theories [10, 11, 12, 13, 31] should be understood as a kinematical effect. The confining properties we have discussed here correspond, on the contrary, to theories in the confining 
phase with dynamical confinement. Namely, we are studying string theory backgrounds believed to be dual to embeddings of $\mathcal{N}=1$ SYM into string theory in such a way that the dynamical confining properties remain. Thus, our calculation are expected to be more than a kinematical effect implied by the strict $N \rightarrow \infty$ limit.

In this paper we have derived the density of states of a string theory dual of hadronic states. Since the string theory is exactly soluble we have been able to extract this quantity exactly. The physical input needed to obtained the solvable limit (large $U(1)$ charge) shows itself in the final answer for the density of states (2.8) by modifying the effective tension to be $\tilde{T}_{s}=T_{s} / J$.

In the second part of the paper, motivated by the exact calculations performed in the first part, we proposed a semiclassical evaluation of the finite temperature partition function. Our proposal is particularly useful for supergravity backgrounds dual to confining theories. We carried this semiclassical calculation for the background of $N$ D5 wrapped on $S^{2}$ whose low energy sector contains pure $\mathcal{N}=1 \mathrm{SYM}[16$. We showed that generically it gives a Hagedorn density of states with the coefficient completely determined by the gauge theory string tension $T_{s}$ (4.21). Interestingly, this is precisely the tension $T_{s}$ of the quark-antiquark potential as calculated in the AdS/CFT framework.

One possible direction is to apply our proposal to other confining theories, most naturally to the embedding of $\mathcal{N}=1$ discussed in [15]. It would also be interesting to study confining theories whose supergravity duals are related to manifolds of $G_{2}$ holonomy. Finally, for the case of nonconfining theories our proposal should lead to string theoretic corrections to the Bekenstein-Hawking entropy.

\section{Acknowledgments}

We would like to thank R. Brower, A. Buchel, J. Gomis, C. Nùnez, E. Rabinovici, G. Semenoff, J. Sonnenschein, M. Strassler and A. Tseytlin. We are especially thankful to D. Kutasov for raising the question of a semiclassical estimate for the Hagedorn density of states in confining theories and to I. Klebanov for a very illuminating discussion. D.V. would like to thank the organizers of the QCD and Strings workshop and the Michigan Center for Theoretical Physics for hospitality during the initial stages of this work.

The research of L.P.Z. is partially supported by the U.S. Department of Energy, while D.V. is supported by DOE grant DE-FG02-91ER40671.

\section{References}

[1] J. Maldacena, Adv. Theor. Math. Phys. 2 (1998) 231 hep-th/9711200.

[2] I. R. Klebanov, Nucl. Phys. B 496 (1997) 231 arXiv:hep-th/9702076.

I. R. Klebanov and A. A. Tseytlin, Nucl. Phys. B 475 (1996) 164 arXiv:hep-th/9604089.

S. S. Gubser, I. R. Klebanov and A. W. Peet, Phys. Rev. D 54 (1996) 3915 arXiv:hep-th/9602135. 
S. S. Gubser, I. R. Klebanov and A. A. Tseytlin, Nucl. Phys. B 499 (1997) 217 arXiv:hep-th/9703040.

S. S. Gubser, I. R. Klebanov and A. A. Tseytlin, Nucl. Phys. B 534 (1998) 202 arXiv:hep-th/9805156.

[3] A. Buchel, Nucl. Phys. B 600 (2001) 219 arXiv:hep-th/0011146.

A. Buchel, C. P. Herzog, I. R. Klebanov, L. A. Pando Zayas and A. A. Tseytlin, JHEP 0104 (2001) 033 arXiv:hep-th/0102105.

S. S. Gubser, C. P. Herzog, I. R. Klebanov and A. A. Tseytlin, JHEP 0105 (2001) 028 arXiv:hep-th/0102172.

[4] A. Buchel and A. R. Frey, Phys. Rev. D 64 (2001) 064007 arXiv:hep-th/0103022.

S. S. Gubser, A. A. Tseytlin and M. S. Volkov, JHEP 0109 (2001) 017 arXiv:hep-th/0108205.

[5] D. Berenstein, J. M. Maldacena and H. Nastase, JHEP 0204 (2002) 013 arXiv:hep-th/0202021.

[6] S. S. Gubser, I. R. Klebanov and A. M. Polyakov, Nucl. Phys. B 636 (2002) 99 arXiv:hep-th/0204051.

[7] J. Polchinski and M. J. Strassler, Phys. Rev. Lett. 88 (2002) 031601 arXiv:hep-th/0109174.

J. Polchinski and M. J. Strassler, arXiv:hep-th/0209211.

[8] R. Hagedorn, Nuovo Cim. Suppl. 3 (1965) 147.

[9] R. R. Metsaev, Nucl. Phys. B 625 (2002) 70 arXiv:hep-th/0112044.

R. R. Metsaev and A. A. Tseytlin, Phys. Rev. D 65 (2002) 126004 arXiv:hep-th/0202109.

[10] L. A. Pando Zayas and D. Vaman, Phys. Rev. D 67 (2003) 106006 arXiv:hep-th/0208066.

[11] B. R. Greene, K. Schalm and G. Shiu, Nucl. Phys. B 652 (2003) 105 arXiv:hep-th/0208163.

R. C. Brower, D. A. Lowe and C. I. Tan, Nucl. Phys. B 652 (2003) 127 arXiv:hep-th/0211201.

[12] G. Grignani, M. Orselli, G. W. Semenoff and D. Trancanelli, arXiv:hep-th/0301186.

[13] Y. Sugawara, arXiv:hep-th/0301035.

Y. Sugawara, Nucl. Phys. B 650 (2003) 75 arXiv:hep-th/0209145.

S. j. Hyun, J. D. Park and S. H. Yi, arXiv:hep-th/0304239.

[14] S. A. Abel, J. L. Barbon, I. I. Kogan and E. Rabinovici, arXiv:hep-th/9911004.

J. L. Barbon, I. I. Kogan and E. Rabinovici, Nucl. Phys. B 544 (1999) 104 arXiv:hep-th/9809033. 
[15] I. R. Klebanov and M. J. Strassler, JHEP 0008 (2000) 052 arXiv:hep-th/0007191.

[16] J. M. Maldacena and C. Nunez, Phys. Rev. Lett. 86 (2001) 588 arXiv:hep-th/0008001.

[17] E. G. Gimon, L. A. Pando Zayas, J. Sonnenschein and M. J. Strassler, JHEP 05 (2003) 039 arXiv:hep-th/0212061.

[18] J. Polchinski, Commun. Math. Phys. 104 (1986) 37.

[19] B. McClain and B. D. Roth, Commun. Math. Phys. 111 (1987) 539.

[20] J. J. Atick and E. Witten, Nucl. Phys. B 310 (1988) 291.

[21] P. Di Francesco, P. Mathieu and D. Sénéchal, Conformal Field Theory, Springer, 1997.

[22] K. H. O'Brien and C. I. Tan, Phys. Rev. D 36 (1987) 1184.

[23] J. G. Russo and A. A. Tseytlin, JHEP 0204 (2002) 021 arXiv:hep-th/0202179.

[24] J. Sonnenschein, arXiv:hep-th/0009146.

Y. Kinar, E. Schreiber and J. Sonnenschein, Nucl. Phys. B 566 (2000) 103 arXiv:hep-th/9811192.

Y. Kinar, E. Schreiber, J. Sonnenschein and N. Weiss, Nucl. Phys. B 583 (2000) 76 arXiv:hep-th/9911123.

[25] J. M. Maldacena, Phys. Rev. Lett. 80 (1998) 4859 arXiv:hep-th/9803002.

S. J. Rey and J. T. Yee, Eur. Phys. J. C 22 (2001) 379 arXiv:hep-th/9803001.

[26] A. H. Chamseddine and M. S. Volkov, Phys. Rev. Lett. 79 (1997) 3343 arXiv:hep-th/9707176.

[27] R. Minasian and D. Tsimpis, Nucl. Phys. B 572 (2000) 499 arXiv:hep-th/9911042.

[28] N. Drukker, D. J. Gross and A. A. Tseytlin, JHEP 0004, 021 (2000) arXiv:hep-th/0001204.

[29] S. Carlip, Phys. Lett. B 186, 141 (1987).

[30] E. Witten, Adv. Theor. Math. Phys. 2 (1998) 505 arXiv:hep-th/9803131.

[31] A. Buchel and L. A. Pando Zayas, arXiv:hep-th/0305179. 